\title{
Nutritional Rickets
}

National Cancer Institute

\section{Source}

National Cancer Institute. Nutritional Rickets. NCI Thesaurus. Code C131447.

Rickets due to dietary deficiency of calcium, phosphate, or vitamin D. 